In Crescendo, 2019; 10(3): 519-535

Fecha de recepción: 06/11/2018

Fecha de aceptación: 20/09/2019

\title{
PROGRAMA DE ENSEÑANZA DE LA TÉCNICA DEL FÚTBOL A TRAVÉS DEL APRENDIZAJE SIGNIFICATIVO EN FUTBOLISTAS PRE JUVENILES
}

\author{
TEACHING PROGRAM OF FOOTBALL TECHNIQUE THROUGH \\ SIGNIFICANT LEARNING IN PRE-YOUTH FOOTBALL PLAYERS
}

\section{Luis Gonzalo Flores Contreras ${ }^{1}$}

\section{RESUMEN}

La presente investigación con enfoque cuantitativo y alcance descriptivo pretende dar una explicación detallada sobre un programa de enseñanza de la técnica del fútbol en el marco del Aprendizaje Significativo en futbolistas prejuveniles de la Escuela de formación deportiva "PUMAS FC" de Pamplona en miras de explicar el tránsito entre lo teórico y lo práctico, fundamentales para la formación integral del deportista. El diseño se desarrolló con una organización de ocho talleres teórico-prácticos, cuya estructura se organizó con situaciones de juego en contexto, que establecidas para el abordaje de los conceptos y la construcción de las definiciones para ser ejecutado en sesiones de entrenamiento, acompañadas de la fundamentación teórica sobre la técnica del fútbol, desde diferentes formas de organización para su ejecución; este diseño busca lograr mejoras en el nivel de conocimientos de los sujetos participantes, dado que se evidenciaron debilidades en los presaberes determinados en la prueba inicial.

El análisis de la información se realizó a través de instrumentos como el cuestionario de actitudes y motivaciones hacia el fútbol y la prueba inicial de conocimientos previos. En conclusión se obtuvo que las situaciones planteadas en los talleres se consideran apropiadas para avanzar en los desempeños de los deportistas prejuveniles, porque permiten mejorar el nivel de conoci-

1 Universidad de Pamplona (Colombia). 
miento y generar habilidades para aprender los distintos controles del balón, la técnica del cabeceo para pasar, conducir y rematar correctamente, el control de movimientos con el muslo y con el pecho, hacer pases con precisión y de forma instantánea, capacidad de recepcionar y de hacer intercambio de pases, a partir de su dominio conceptual.

PALABRAS CLAVE: Programa de enseñanza, fútbol, proceso de aprendizaje, prejuveniles, situaciones de juego.

\section{ABSTRACT}

The present research with a quantitative approach and descriptive scope is intended to provide a detailed explanation of a soccer technique teaching program in the framework of Significant Learning in pre-youth soccer players of the PUMAS FC School of Sports Training in Pamplona in order to explain the transit between theoretical and practical, fundamental for the integral training of the athlete. The design was developed with an organization of eight theoretical and practical workshops, whose structure was organized with game situations in context, which were established to address the concepts and the construction of the definitions to be executed in training sessions, accompanied by the theoretical foundation on the technique of football, from different forms of organization for its execution; This design seeks to achieve improvements in the level of knowledge of the participating subjects, given that there were weaknesses in the knowledge determined in the initial test.

The analysis of the information was carried out through instruments such as the questionnaire of attitudes and motivations towards football and the initial test of previous knowledge. In conclusion it was obtained that the situations raised in the workshops are considered appropriate to advance the performance of pre-youth athletes, because they allow to improve the level of knowledge and generate skills to learn the different controls of the ball, the pitching technique to pass, drive and to finish off correctly, the control of movements with the thigh and with the chest, make passes with precision and instantaneously, ability to receive and make exchange of passes, from its conceptual domain.

KEY WORDS: Teaching program, football, learning process, youth, game situations.

\section{INTRODUCCIÓN}

En diversas investigaciones "se han tratado aspectos relacionados con la pedagogía y didáctica del fútbol infantil y juvenil”, los que tienen ver con la didáctica del juego, pedagogía del fútbol, las etapas del desarrollo del futbolista, aspectos metodológicos del futbol, el entrenador del fútbol. Pero, existe muy poca literatura que contempla el desarrollo cognitivo del joven deportista o el nivel de conocimiento sobre la técnica del futbol, que le permita tener "una visión amplia e integradora sobre el desempeño y la formación integral en este deporte". 
"Tener en cuenta estos aspectos se considera fundamental para que el entrenador pueda plantear sesiones prácticas de entrenamiento, acorde con los intereses psicológicos de los participantes, respetando el nivel de desarrollo biológico y cognitivo de los mismos y a su vez le permite maximizar su comportamiento competitivo (entiéndase esto como el desarrollo de las áreas técnico-táctica, física y volitiva) (Rivas, 2012)".

En el trabajo de investigación se presenta un programa de enseñanza sobre el objeto de conocimiento La Técnica del fútbol, orientada bajo los lineamientos del Aprendizaje Significativo utilizando como eje fundamental para su diseño la fundamentación teórica de la disciplina deportiva, en miras al fortalecimiento de los procesos de aprendizaje, con prejuveniles de la Escuela de formación deportiva "PUMAS FC" de la ciudad de Pamplona, dada la importancia en el desarrollo de competencias que el manejo teórico puede establecer a partir de situaciones específicas cuyos escenarios sean los adecuados a fenómenos de juego presentes en la vida real del deporte.

En las intervenciones planteadas los participantes tendrán la oportunidad de manipular elementos conceptuales, ejecutar y comprobar bajo ciertos contextos, aspectos que los llevaron hacia la comprensión de conceptos asociados al tema objeto de estudio la técnica del fútbol, desde diferentes ópticas de razonamiento, en la construcción de su propio conocimiento.

Según Casal (2003), se pretende partir del conocimiento de las características, finalidades y objetivos de la técnica del fútbol, y de conocer a los jugadores, sus rasgos característicos, capacidades y habilidades en los diferentes momentos del proceso de aprendizaje. En este caso, se prevé estar determinados por el nivel de conocimiento y desarrollo cognitivo en los escenarios que se presenten. Sin una adecuada comprensión de estos aspectos no se podría obtener utilidad y ventajas en el diseño y enfoque de una determinada metodología de enseñanza.

Ahora bien, el aprendizaje y la enseñanza de los deportes grupales o colectivos, como objeto del conocimiento, se ha constituido un tema de interés e investigación desde hace algunas décadas; en estos estudios se han hecho comparaciones sobre el efecto de los modelos teóricos más utilizados en la enseñanza deportiva: se contrasta el enfoque tradicional o también llamado técnico versus el enfoque alternativo o el que se fundamenta en la técnica.

$\mathrm{Al}$ respecto, algunos autores plantean que el producto del proceso de racionalización de la sociedad industrial y el "falso silogismo", conocido como modelo 
tradicional, viene concentrándose en la técnica deportiva y en la preparación física, bajo el supuesto de que "los medios y métodos que incrementan el rendimiento en los deportes individuales logran el mismo objetivo en los deportes de equipo" (Martínez, 2010).

Así mismo, plantean que el modelo que se fundamenta en la técnica presenta dificultades y muchas limitaciones, por ello se pretende superar las desventajas de la concepción tecnicista, fundamentando el componente técnico, con situaciones reales de juego, estrategias, juegos individuales y colectivos, otorgando un lugar importante a los procesos cognitivos y al nivel de conocimiento implicados en la acción de los deportistas.

Se resalta la necesidad de analizar las condiciones de la mediación para promover las competencias que debe tener un jugador prejuvenil en los deportes grupales, como es el caso del futbol, a partir de la experimentación técnica con el entorno real de juego apoyándose en situaciones significativas para los jóvenes. Caso particular es el de la Escuela de Formación de Fútbol PUMAS de la ciudad de Pamplona donde se presentan fuertes debilidades en los jóvenes de 13 a 15 años en el trabajo específico sobre elementos propios de la técnica, el dominio del balón, el cabeceo, el pase, el engaño, con trabajo en equipo sobre situaciones de juego en la búsqueda para conseguir el objetivo que es el gol.

El dominio y la conducción del balón en los jóvenes se puede emplear para conducir la pelota y regatear o driblar a los contrarios, así como para pasar el balón a los compañeros, recepción con el pecho y cabeceo, patear a la portería; se convierten en elementos técnicos fundamentales para jugar el fútbol y la competencia principal que se debe desarrollar en los deportistas pre-juveniles; en las Escuelas de Formación de Fútbol de la ciudad de pamplona, las prácticas de entrenamiento no han repercutido adecuadamente en el desarrollo de las potencialidades que se requieren, se observa que existe un alto porcentaje de deficiencias en destrezas motrices.

Desde esta perspectiva, esta investigación de tipo cuantitativo proyectada para ser aplicada, evaluada y analizada en un año, se plantea como una propuesta didáctica de trabajo, conformada por una serie de talleres diseñados bajo el modelo teórico del Aprendizaje Significativo que busca destacar los conocimientos de la técnica del fútbol, y que implica la utilización de estrategias y procedimientos que abordan tanto los tipos de aprendizaje que se producen en el campo de juego o en el juego real, como a las características y rasgos psicológicos propios de los 
jugadores que ponen en juego cuando aprenden; la finalidad es clarificar las maneras de llevar a cabo el aprendizaje de los elementos técnicos de este deporte, determinando las formas de aprendizaje que usan los jugadores al realizar actividades propias del fútbol que se les proponen en situaciones reales de juego y establecer sus condiciones en el desarrollo de sus capacidades cognitivas.

Para ello se establece que es conveniente conocer los saberes previos, el nivel y el tipo de razonamiento que emplea el joven deportista para así poder crear experiencias de aprendizaje que permitan desarrollar su conocimiento en esta disciplina, teniendo como punto de partida sus procesos cognitivos y el nivel de razonamiento en el que se encuentra cada uno de ellos (Acosta, 2017).

A partir de lo expuesto surgen interrogantes como ¿Qué fundamentos teóricos y metodológicos de la enseñanza técnica del Fútbol tienen los jóvenes de la Escuela de formación de fútbol PUMAS de Pamplona?, ¿Cuál es el nivel de conocimientos sobre la técnica del fútbol de estos deportistas?, ¿El aprendizaje significativo sirve como fundamento teórico para diseñar un programa de enseñanza de la técnica del fútbol?, ¿Cómo se puede diseñar un programa de enseñanza de la técnica del Fútbol con el Aprendizaje Significativo?

\section{Marco Teórico}

\section{El proceso de aprendizaje de los estudiantes}

El proceso de aprendizaje formal implica el cumplimiento de varias fases entrelazadas entre sí: motivación, interés, atención, adquisición, comprensión, asimilación, aplicación, transferencia y evaluación (Yáñez, 2016). Este trabajo se centró en un tema específico: la técnica del futbol utilizando su fundamentación teórica como eje central y enmarcada en el Aprendizaje Significativo; con el fin de describir en forma detallada el diseño de un programa de enseñanza de la técnica del fútbol en futbolistas prejuveniles de la Escuela de formación deportiva "PUMAS FC" de Pamplona en miras de explicar el tránsito entre lo teórico y lo práctico fundamentales para la formación integral del deportista.

Para Shunk (2012), "Aprender implica construir y modificar nuestro conocimiento, así como nuestras habilidades, estrategias, creencias, actitudes y conductas." (p.3). Ormrod (2005) citado en García (2012) considera que: "el aprendizaje es el medio mediante el que no solo adquirimos habilidades y conocimiento, sino también valores, actitudes y reacciones emocionales, cuando se produce 
aprendizaje se realiza un cambio permanente en las diferentes relaciones y asociaciones mentales que resultan de la experiencia adquirida.

\section{El aprendizaje en el constructivismo}

El constructivismo es un enfoque educativo que supone un esfuerzo por integrar diversas teorías psicológicas del aprendizaje y la epistemología de la construcción de conocimientos (Pozo, 1989; Carretero, 1994 citado en Acosta, 2017). En esta teoría se destaca el papel de los conceptos, las características y relaciones entre ellos; aquí se privilegian los conocimientos previos, los presaberes y el lenguaje que permite dar forma, codificar, y adquirir nuevos significados.

La epistemología de la construcción de conocimientos, la interacción entre el pensamiento y la realidad es la fuente del conocimiento, los que se van adquiriendo no son los últimos ni los definitivos, sino que su proceso de formación sigue un dinámica real por la que el conocimiento anterior ejerce influencia en el presente o actual y este a su vez interviene en el conocimiento futuro, es por esto que lo adquirido, por muy universal o verdadero que sea, representa solo una perspectiva de la realidad.

Para el constructivismo el aprendizaje es un proceso activo y dinámico, fruto de una construcción personal, en la que intervienen e influyen otros factores como la enseñanza (contenidos, metodología, recursos, evaluación) y los contextos culturales, que se constituyen como partes fundamentales para la construcción. En este sentido, se considera que el desarrollo personal, el desarrollo intelectual, el aprendizaje y la educación son procesos que se encuentran profundamente interrelacionados.

La teoría del constructivismo se apoya en modelos de desarrollo, que resaltan la dimensión activa y dinámica del que aprende en el proceso de enseñanza y aprendizaje, partiendo del hecho que es necesario aprender conceptos, pero también es fundamental aprender procedimientos y desarrollar actitudes. En diversas investigaciones se establece que la construcción de nuevos conocimientos es la principal forma de aprender significativamente, porque, realmente, lo que se busca y se consigue es crear nuevos significados.

\section{El constructivismo en la Escuela de formación de futbol de Pamplona}

La Escuela de formación de futbol "PUMAS FC" de Pamplona fundamenta sus bases pedagógicas en el modelo constructivista donde menciona algunos teó- 
ricos como Piaget, David Ausubel, Lev Vygotsky, Maslow, Roger, Frank, Feuerstein y Subiría. En este sentido lo describe como el modelo que pretende la formación de personas como sujetos activos, capaces de tomar decisiones y emitir juicios de valor, lo que implica la participación de profesores y estudiantes que interactúan en el desarrollo de la clase para construir, crear, facilitar, liberar, preguntar, criticar y reflexionar sobre la comprensión de las estructuras profundas del conocimiento deportivo.

El centro de este modelo es el aprender haciendo, donde el maestro es un facilitador que contribuye al desarrollo de capacidades de los estudiantes para pensar, idear, crear y reflexionar. El objetivo de la escuela de fútbol es desarrollar las habilidades del pensamiento de los individuos de modo que ellos puedan progresar, evolucionar secuencialmente en las estructuras cognitivas para acceder a conocimientos cada vez más elaborados y potencializar sus capacidades motoras en procura de un buen desempeño deportivo.

\section{Aprendizaje Significativo}

Se ha tomado como referencia teórica para este estudio el Aprendizaje Significativo, que se considera una importante teoría psicológica y educativa desarrollada por Ausubel y sus colaboradores que profundiza en el significado y sentido del aprendizaje (Ausubel, Novak \& Hanesian, 1983 citado en Acosta, 2017). Se constituye como una teoría psicológica porque se centra en los procesos propios del sujeto cuando aprende, haciendo énfasis en todo lo que sucede en el aula cuando los estudiantes aprenden; en la naturaleza de ese aprendizaje; en las condiciones que se requieren para que este se produzca; en sus resultados y, consecuentemente, en su evaluación (Ausubel, 1976).

Para Pozo (1989) citado en Ortuzar (2011) la teoría del Aprendizaje Significativo se constituye como una teoría cognitiva de reestructuración; se trata de una teoría psicológica que se gesta y se construye bajo la orientación de un enfoque organicista del sujeto y que está basado y centrado en el aprendizaje generado en un contexto escolar. En la teoría de Ausubel se da gran importancia al hecho de diferenciar el aprendizaje mecánico o memorístico del aprendizaje significativo; cuando el sujeto, no establece relación o conexión, con los conceptos que posee en su estructura cognitiva al enfrentarse a nuevos conocimientos, se considera que aprende de forma memorística. Por el contrario, si es capaz de relacionar la nueva información con las ideas o conceptos previos que posee en su estructura mental, entonces está apren- 
diendo de manera significativa, y esa es la intencionalidad directa de las actividades que hemos seleccionado para este estudio (p. 39).

$\mathrm{El}$ aprendizaje significativo es un proceso que requiere una serie de condiciones o requisitos establecidos por Ausubel (1996) citado en Chrobak (2017) en su teoría, los cuales se mencionan a continuación y fueron considerados para esta investigación: (a) El estudiante debe contener ideas o conceptos para incluir en su estructura cognitiva, de tal forma que este tipo de conceptos con significados más concretos permitan entender y comprender la nueva información dándole significado y sentido; (b) Las situaciones de aula demuestran que es fundamental que el estudiante tenga una actitud o disposición favorable para aprender de manera significativa; es decir, el alumno debe estar motivado y dispuesto a relacionar el conocimiento nuevo que está aprendiendo con lo que ya conoce, esto con el fin de modificar los esquemas de conocimiento; (c) Del significado lógico al psicológico. El significado lógico que corresponde a la estructura científica propia de la materia debe convertirse en significado psicológico, el que alcanza una persona cuando asimila un significado lógico dentro de su propia estructura mental, que se consigue con la ayuda de los conceptos que pueden ser incluidos; y (d) Contenidos potencialmente significativos. El material presentado debe poseer significado para el sujeto que aprende, sus elementos deben estar relacionados y estructurados entre sí. Dependiendo de la organización del nuevo conocimiento, de su claridad y estabilidad, será mejor su acomodación y su retención en la estructura mental del individuo.

\section{Actitudes para el aprendizaje de los deportes}

La actitud positiva hacia el aprendizaje de las disciplinas deportivas es la base que mueve toda conducta, y permite provocar cambios tanto a nivel escolar y en otros ámbitos; es el elemento primordial para aumentar el desempeño de los jóvenes. Según Tapia (1991) citado en Acosta (2017) hace parte de la motivación, que es un factor relevante que conlleva el éxito en cualquier área. Este autor afirma que: "querer aprender y saber son las condiciones personales básicas que permiten la adquisición de nuevos conocimientos y la aplicación de lo aprendido de forma efectiva cuando se necesita".

Para (Nieves 1993 citado en Meza, 2012), "las actitudes hacia el aprendizaje de una disciplina influyen, en el tiempo y el esfuerzo dedicados a trabajar cuestiones relativas a esa asignatura y esto, a su vez repercute en el rendimiento, la nota obtenida y una actitud positiva facilita el aprendizaje mientras que una actitud 
negativa lo dificulta" (p. 20). Otros autores como (Del Puerto \& Minnaard, 2003; García, 2011), afirman que las actitudes hacia los deportes influyen en el aprendizaje y consideran que los alumnos con actitudes positivas obtienen generalmente logros superiores a los que alcanzan los alumnos con actitudes negativas; del mismo modo, un alumno con facilidad para estas disciplinas disfrutará más que aquel que tiene problemas en su estudio (p. 67).

Con referencia a lo anterior, para que un estudiante en este caso un deportista obtenga un aprendizaje debe existir por parte de él buena disposición e interés, el rol del docente entrenador en cuanto a la motivación antes, durante y después del aprendizaje y la estimulación de las acciones logradas por el estudiante marcaran un ambiente positivo hacia la construcción del conocimiento.

\section{Fundamentos Técnicos del Fútbol Concepto de Técnica}

"Según Rivas (2012) existen variadas definiciones acerca del concepto de técnica futbolística, no obstante, que la técnica debe ser orientada de tal manera que cumpla las exigencias de ser económica y de una movilidad rápida". "Por otro lado, se hace manifiesto que la técnica se concibe como el modo de ejecutar todos los movimientos posibles en el fútbol". "Para el caso que nos ocupa, se tomará la técnica como todos aquellos movimientos o acciones con y sin balón que realiza el futbolista durante la ejecución y que dichas acciones se realizan para tener dominio del balón cuya finalidad es dirigirlo al arco contrario".

\section{"Pase con el pie o Toques del balón"}

"Se define como el lanzamiento o la dirección que se le da al balón conscientemente con alguna parte del pie y se considera el elemento técnico básico del juego el cual es fundamental en su aprendizaje y enseñanza en la etapa juvenil". "Es la principal destreza del jugador tanto para ataque como para defensa, el cual permite conservar colectivamente el balón, preparar los ataques, invertir el juego, contraatacar, dar el pase a gol o último pase".

"Se pueden clasificar los pases con el pie por la parte del pie que contacta el balón y por la transmisión de la fuerza con algunas partes de la pierna; según esto el balón se puede tocar con el borde interno del pie, el borde exterior del pie, el empeine exterior, el empeine interior, la puntera, la rodilla y el talón”.

"En la utilización táctica de los toques se pueden clasificar en: pase, remate balón cruzado, centro, saque de puerta, entre otros, por lo tanto, se puede afirmar 
entonces que la utilización de un toque determinado está regulada por la situación táctica del momento". "En el caso de la altura del balón, existen tres toques o movimientos de contacto balón a ras del suelo, de bote pronto y balón alto o por aire”.

"En su aspecto técnico, el deporte del fútbol sugiere algunos posibles movimientos corporales específicos (Howe, 1991 citado en Rivas, 2012) como determinar con exactitud el tipo de toque más adecuado a utilizar dependiendo de la situación, colocar el pie de apoyo siempre al lado del balón, y la puntera del zapato deberá estar dirigida hacia la dirección donde se quiere enviar balón; así mismo la pierna que se va a realizar el contacto con el balón, deberá extenderse hacia atrás dependiendo del grado de fuerza que se le quiera imprimir al toque, la planta del pie debe estar extendida (la punta del pie mira hacia abajo) fuertemente". "Esto es aplicable a todos los toques del balón, a excepción del toque con el borde interno y en el toque del balón propiamente dicho, el grado de fuerza a aplicar y le toque a utilizar, dependerá exclusivamente de la situación de juego y el objetivo del futbolista".

\section{Borde interno y Borde externo}

"Este toque utiliza la cara interna del pie, y abarca desde el inicio del dedo "gordo", pasa por el tobillo interno, hasta el hueso calcáneo". "La ventaja que brinda dicha técnica es que posee una mayor superficie de contacto del pie con el balón, lo que la constituye una de las técnicas relativamente más fáciles respecto a su manejo".

"Para Albún, (1993) citado en Rivas, (2012) el borde externo es un recurso poco utilizado por el jugador, dado que su técnica tiene un grado de dificultad, no brinda tanta seguridad y precisión en el pase, pero es una muy estética". "La superficie de contacto comprende toda la cara externa del pie, es decir; desde el dedo "pequeño", parte exterior del tobillo, hasta el hueso calcáneo; esta técnica es utilizada para realizar pases cortos o hacer devoluciones en el juego de pared".

\section{Conducción de balón}

"Para Rivas (2012) es la rodadura y avance del balón utilizando cualquier parte del pie, y sobre el terreno de juego". "Es la maniobra de avanzar con el balón en los pies, es el dominio y desplazamiento con el balón, mediante una serie de toques con cualquier parte del pie". "En determinadas situaciones del partido, se debe recurrir a la conducción de tal forma que le permita encontrar una opción clara de pase o bien un remate". 
"Esta acción técnica se necesita cuando se presenta el contraataque, donde el jugador deberá conducir el balón a máxima velocidad, sobre todo en los últimos sectores de la cancha". "Por otro lado, en el partido ha de emplearse incondicionalmente en diversas situaciones, lo que lo hace un elemento importante en el proceso de enseñanza y aprendizaje de la técnica de este deporte". "Se trata de un medio de progresión individual y desarrollo de esta competencia en un espacio libre". "Para conducir adecuadamente el balón implica tener dominio en todo momento, lo que indica un buen equilibrio y un excelente manejo de los apoyos".

"Dentro de las recomendaciones técnicas de su ejecución se relaciona que durante la acción técnica es necesario llevar la vista al frente, de tal forma, que no se pierda la panorámica de juego". "Sin embargo, en jugadores principiantes esta condición no es tan necesaria; para lograr una mayor eficacia, es recomendable la utilización de otros recursos técnicos complementarios como la finta y regate; se debe conducir solo lo necesario, pues normalmente en este tipo de acciones técnicas son muy frecuentes las lesiones, en determinadas situaciones de juego es aconsejable mantener un ritmo en la conducción, sin embargo cuando se va a enfrentar a rivales es recomendable el cambio de ritmo y dirección como recurso para desconcentrar al rival (Rivas, 2012)”.

\section{DISEÑO METODOLÓGICO}

Para este proyecto de investigación se tomó el enfoque cuantitativo que utiliza la recolección de datos para probar hipótesis con base en la medición numérica y el análisis estadístico, con el fin establecer pautas de comportamiento y probar teorías (Hernández, 2016), puesto que con él, se refleja la necesidad de medir y estimar las magnitudes del fenómeno de estudio denominado la técnica del fútbol, se plantea un problema de estudio delimitado y concreto, sus preguntas de investigación son sobre cuestiones específicas, se hace revisión de la literatura y construcción del marco teórico como orientador del proceso de investigación.

El alcance es de tipo descriptivo dado que se pretende explicar en detalle la realidad de situaciones, contextos, fenómenos, eventos y sucesos, con el fin de especificar cómo suceden, cómo son y cómo se manifiestan; se miden las variables nivel de conocimiento de la técnica del fútbol, motivaciones, actitudes y comportamientos hacia esta disciplina deportiva, metodologías de enseñanza y desempeño del docente entrenador en las sesiones de trabajo. Se pretende recoger los datos, exponer y resumir la información de manera cuidadosa y luego analizarla 
minuciosamente, a fin de extraer generalizaciones significativas que contribuyan al conocimiento.

\section{Las variables}

En Arias (2012) se señala que una variable es una característica o cualidad, magnitud o cantidad susceptible de sufrir cambios y es objeto de análisis, medición, manipulación o control en una investigación. Las variables identificadas en el presente estudio indican en forma directa lo que se observa o se mide, estableciendo en estos aspectos su importancia.

Para esta investigación, la definición conceptual de la variable Nivel de conocimiento de la técnica del fútbol es: avance en la producción del saber sobre la técnica del fútbol y representa un incremento en la complejidad con que se explica o comprende las situaciones de juego; y de la variable Programa de enseñanza del fútbol es: herramienta educativa que facilita el aprendizaje del fútbol de una manera adecuada y eficaz. De igual manera se definen otras variables para este estudio como son Explicaciones del docente, Planificación de clase, Motivaciones del estudiante y Aprendizaje colaborativo de acuerdo a la intencionalidad del trabajo, al planteamiento de los problemas y a los objetivos establecidos.

\section{Población}

La población corresponde a los sujetos o participantes que intervienen en la investigación, Fracica (1988 citado por Bernal, 2010) define la población como "el conjunto de todos los elementos a los cuales se refiere la investigación. La población objeto de estudio en esta investigación corresponde a los 20 jóvenes deportistas con edades comprendidas entre 13 y 15 años de la Escuela de Formación Deportiva "PUMAS FC" de la ciudad de Pamplona.

Selección de la muestra: para el caso de este estudio no se realizó muestreo probabilístico, dado que se hizo la aplicación con el total de sujetos pertenecientes a la escuela de formación de fútbol "PUMAS FC" por cuanto la muestra es muy pequeña y teniendo en cuenta que cumplieran con los criterios de inclusión. La muestra para este estudio fue en total de 20 jóvenes deportistas de la Escuela de Formación Deportiva "PUMAS FC" de la ciudad de Pamplona.

\section{Operacionalización de la variable}

Se define como el paso de una variable teórica a indicadores empíricos verificables y medibles e ítems o equivalentes (Solís, 2013 citado en Hernández, 2016). 
Para poder realizar la operacionalización de la variable se debe hacer fundamentado en la definición conceptual de ella misma; esta organización hace más fácil la construcción de un instrumento para recolección de información, que será producto del análisis y desglose de la variable en sus dimensiones o componentes, luego a los indicadores y finalmente a los ítems o reactivos y sus categorías. Las variables tenidas en cuenta para este estudio son Explicaciones del docente, Planificación de clase, Motivaciones del estudiante y Aprendizaje colaborativo.

Estas variables se organizaron y analizaron de acuerdo a sus respectivas dimensiones, indicadores e ítems, generados de acuerdo a la intencionalidad del trabajo, a la revisión del estado del arte del objeto de estudio, a la metodología y a los objetivos establecidos.

\section{Instrumentos de Recolección de datos}

Un buen instrumento de medición es aquel que permita registrar datos observables que representan verdaderamente los conceptos o las variables que el investigador tiene en mente (Grinnell, Williams y Unrau, 2009 citados en Hernández, 2016). Otros autores lo señalan como la función de la medición es establecer una correspondencia entre el "mundo real" y el "mundo conceptual", lo importante es que represente lo que realmente se quiere describir.

En investigación se disponen de varios tipos de instrumentos para medir las variables de interés y en algunos casos llegan a combinarse varias técnicas de recolección de los datos. En el enfoque cuantitativo siempre se aplican instrumentos para medir las variables; Para este estudio se utilizaron varios instrumentos de medición como el cuestionario de actitudes y motivaciones hacia el fútbol, la prueba inicial de conocimientos previos y los Talleres sobre la técnica del fútbol los cuales se describen a continuación:

El Cuestionario de actitudes y motivaciones hacia el fútbol, se diseñó de 22 ítems con escala tipo Likert (Nunca, Raras veces, A veces, A menudo, Siempre); consiste en un conjunto de cuestiones presentadas, en forma de afirmaciones o juicios, ante los cuales se pide la reacción de los participantes. Se presenta cada afirmación y se solicita al sujeto que exprese su opinión escogiendo uno de los cinco puntos o categorías de la escala. A cada punto se le asigna un valor numérico, el participante obtiene una puntuación respecto de la afirmación y al final su puntuación total, sumando las puntuaciones obtenidas en relación con todas las afirmaciones (Hernández, 2016). 
La prueba inicial de conocimientos previos o prueba diagnóstica fue diseñada para verificar los saberes y pre saberes que los participantes tenían acerca de la técnica del fútbol. Las ocho primeras preguntas corresponden a preguntas cerradas de selección múltiple con única y múltiple respuesta; los tres últimos ítems fueron de tipo abierto puesto que proporcionan una información más amplia y son particularmente útiles cuando no tenemos información sobre las posibles respuestas de las personas o la que tenemos es insuficiente (Phillips, Phillips y Aaron, 2013, citados en Hernández, 2017), y cuyas conclusiones sirvieron como punto de partida para el diseño de las estrategias pedagógicas pertinentes.

Talleres para la intervención sobre la técnica del fútbol diseñados a partir de las debilidades encontradas en la prueba inicial; fueron estructurados en el marco del aprendizaje significativo, incorporando un resumen teórico del concepto, direcciones electrónicas de videos ilustrativos para complementar la teoría, varias preguntas tipo ICFES de selección múltiple con única respuesta, y ejercicios prácticos para realizar en el campo deportivo sobre la teoría estudiada en la sesión. Las temáticas desarrolladas en estos talleres fueron en su orden: Control dirigido u orientado, El Cabeceo, El Muslo, El Pecho, Control de balón y la conducción, Los Engaños y Control orientado y Pase.

\section{RESULTADOS Y DISCUSIÓN}

Este apartado se centra en la interpretación de los resultados de los métodos de análisis cuantitativo; el análisis de los datos se efectuó sobre la matriz de datos diseñada en un programa computacional, en dos partes fundamentales, una parte definiciones de las variables, Explicaciones del docente, Planificación de clase, Motivaciones del estudiante y Aprendizaje colaborativo de acuerdo a la intencionalidad del trabajo, al planteamiento del problemas y a los objetivos establecidos; y la otra parte, la matriz de datos, con las definiciones que previamente ha preparado el investigador, con los parámetros para la matriz, nombre de cada variable, tipo de variable o ítem, ancho en dígitos, etc.

Propuesta Pedagógica. La Enseñanza de la Técnica del Fútbol a través del Aprendizaje Significativo en Futbolistas Pre juveniles de la Escuela de formación deportiva "PUMAS FC" de la ciudad de Pamplona, es innovadora puesto que contiene elementos con respecto al modelo teórico y al campo disciplinar, que se desarrollan a través de talleres por sesiones que realizan una serie de actividades 
en contextos específicos respondiendo a problemas de aprendizaje del aula, diseñando estrategias para el mejoramiento de los mismos.

Cada sesión de clase se enmarca en los conocimientos previos, las evidencias de aprendizaje y contextos específicos en la búsqueda de la consecución de los objetivos. Posteriormente se plantean talleres los cuales presentan actividades en el marco del Aprendizaje significativo, preguntas tipo pruebas "saber" y situaciones prácticas de juego. Los Talleres contienen entre cuatro o cinco actividades que corresponden a estrategias para el fortalecimiento del aprendizaje de la técnica del fútbol, que es uno de los dominios que presenta el más bajo rendimiento en el desempeño de los deportistas.

\section{CONCLUSIONES}

- A partir de la aplicación de la prueba diagnóstica se logró identificar los pre-saberes y saberes acerca del conocimiento de la técnica del futbol, donde la mayoría de los participantes realizaron actividades tendientes a la verificación de elementos conceptuales específicos de este dominio. Estos resultados fueron tomados como base para el diseño de cada una de las actividades y talleres propios de cada sesión de trabajo, con el objetivo de avanzar en los estudiantes su nivel de conocimiento.

- Se pudo precisar la ubicación de los deportistas de acuerdo con su nivel de conocimiento, a partir de las respuestas obtenidas en la solución del cuestionario sobre la técnica del fútbol. En esta etapa se pudo verificar que los jóvenes no tienen manejo teórico acerca de elementos técnicos como cabeceo, regates o engaños, conducción y dominio del balón, entre otros.

- Por medio del cuestionario de actitudes y motivaciones, se pudo establecer qué concepciones y estímulos tienen los deportistas hacia el fútbol, hacia su enseñanza y su aprendizaje; presentaron fuerte tendencia hacia las variables Explicaciones del docente, planificación de clase, motivaciones del estudiante y trabajo en equipo.

- Con los análisis anteriores se establecieron las temáticas pertinentes, las características y la estructura formal de la propuesta didáctica sobre la técnica del Fútbol a través del Aprendizaje Significativo para el mejoramiento del nivel de conocimiento de los deportistas, teniendo en cuenta 
los ejes conceptuales en los que se evidenciaron mayores debilidades: Control dirigido u orientado, Cabeceo, Muslo, Pecho, Control de balón y la conducción, Engaños, Control orientado y Pase y Arquero.

- Se generó la estrategia pedagógica para mejorar el proceso de aprendizaje de la técnica del futbol en el marco del Aprendizaje Significativo, otorgando la posibilidad de ser abordada de forma deductiva e inductiva a través de situaciones en contexto para observar, clasificar y analizar según las temáticas planteadas.

- Se utilizaron situaciones de juego con variaciones en los tipos de ejercicios, utilizando el fundamento teórico de la técnica del fútbol como medio facilitador del proceso de aprendizaje desde los contextos reales y las diversas posibilidades que ofrece la práctica de este deporte.

- Se recrearon ocho talleres para las sesiones de trabajo denominados Control dirigido u orientado, Cabeceo, Muslo, Pecho, Control de balón y la conducción, Engaños, Control orientado y Pase y Arquero, que contienen actividades diversas en situaciones reales de juego. Se establecieron los parámetros para impulsar el componente actitudinal del estudiante dado que este tipo de trabajos despierta atención, interés, actitud positiva, concentración y autoestima, elementos indispensables para mejorar los niveles de conocimiento en los deportistas.

- Las estrategias pedagógicas recogieron aspectos como el concepto de cada elemento técnico, las características fundamentales, los tipos, clasificaciones y el gesto técnico, con el fin de brindarle el abordaje teórico requerido para el estudio y conocimiento de estos ejes temáticos. Se propició el trabajo en grupo y colaborativo el cual permite que los estudiantes intercambien ideas y propuestas de solución a los diferentes problemas planteados y les genera más confianza en el momento de argumentar sus soluciones ya que así construyen sus conocimientos de una forma más significativa.

- La manipulación de preguntas tipo ICFES busca desarrollar en el estudiante competencias para el análisis de situaciones reales, que propician el logro de habilidades y destrezas teóricas en estos ejes temáticos, y que sirven de refuerzo para la realización de los ejercicios prácticos, en pro de una mejora en la formación integral del deportista. 
- El uso de la fundamentación teórica se constituye como una herramienta importante en el proceso de enseñanza y aprendizaje de la técnica del futbol, dado que le permite al estudiante ejercitarse mentalmente en un ambiente propio de este deporte, por medio de la solución de situaciones específicas del contexto; se fomenta en la estrategia el hecho de debatir y presentar las soluciones a las situaciones planteadas en los talleres que conlleve a aclarar dudas, interpretar mejor el tema y construir conocimiento a partir de las ideas y razonamientos de los compañeros.

- Se concluye que el proyecto Programa de enseñanza de la técnica del fútbol a través del aprendizaje significativo en deportistas prejuveniles se constituye en una propuesta metodológica de carácter innovador, progresista, original y diferente, que pretende generar desarrollos en el nivel de conocimiento de los jóvenes adolescentes.

\section{REFERENCIAS BIBLIOGRÁFICAS}

Acosta, D. (2017). La Función Cuadrática en el marco del Modelo de Van Hiele utilizando Geogebra para el fortalecimiento del proceso de aprendizaje de los estudiantes del grado noveno del Instituto Técnico Municipal los Patios. Universidad Autónoma de Bucaramanga.

Arias, F. (2012). El Proyecto de Investigación Introducción a la metodología científica. 6⿳a Edición Editorial Episteme. ISBN: 980-07-8529-9. Caracas - República Bolivariana de Venezuela.

Ausubel, D. P., Novak, J. D., \& Hanesian, H. (1983). Psicología educativa: un punto de vista cognoscitivo (Vol. 2). México: Trillas.

Bernal, C. (2010). Metodología de la investigación. Bogotá: Editorial Pearson.

Casal, C. (2003). Metodología de la enseñanza del fútbol. Book · January 2003 with 2,417 Reads. ISBN 84-8019-698-X. Publisher: Ed. Paidotribo. Catholic University of Valencia "San Vicente Martir".

Chrobak, R (2017). El aprendizaje significativo para fomentar el pensamiento crítico. Archivos de Ciencias de la Educación. 2017, vol. 11, nro. 12, e031. En Memoria Académica. Disponible en: http://www.memoria.fahce.unlp.edu.ar/art_revistas/pr.8292/pr.8292.pdf.

García, F (2012). La enseñanza y el aprendizaje. Universidad Autónoma de Ciudad Juárez. Año 12, No 57, Especial No 1. CULCyT//Septiembre-Diciembre, 2015.

García, M. (2011). Evolución de actitudes y competencias matemáticas en estudiantes de secundaria al introducir GeoGebra en el aula. Tesis doctoral, Almería, Universidad de Almería

Hernández Sampieri, R., Fernández Collado, C. \& Baptista Lucio, P. (2016). Metodología de la investigación. Sexta edición. México: Editorial Mc Graw Hill. 